\title{
Shielding Synchrotron Light Sources: Advantages of Circular Shield Walls Tunnels
}

\author{
S.L. Kramer \\ Design and Accelerator Operations Consulting \\ 568 Wintergreen $\mathrm{Ct}$ \\ Ridge, NY 11961 \\ and \\ V. J. Ghosh and M. Breitfeller \\ NSLS-II, Brookhaven National Laboratory ${ }^{1}$ \\ Upton, NY 11973 \\ April 19, 2016
}

\section{Abstract}

Third generation high brightness light sources are designed to have low emittance and high current beams, which contribute to higher beam loss rates that will be compensated by Top-Off injection. Shielding for these higher loss rates will be critical to protect the projected higher occupancy factors for the users. Top-Off injection requires a full energy injector, which will demand greater consideration of the potential abnormal beam miss-steering and localized losses that could occur. The high energy electron injection beam produce significantly higher neutron component dose to the experimental floor than lower energy injection and ramped operations. High energy neutrons produced in the forward direction from thin target beam losses are a major component of the dose rate outside the shield walls of the tunnel. The convention has been to provide thicker $90^{\circ}$ ratchet walls to reduce this dose to the beam line users. We present an alternate circular shield wall design, which naturally and cost effectively increases the path length for this forward radiation in the shield wall and thereby substantially decreasing the dose rate for these beam losses. This shield wall design will greatly reduce the dose rate to the users working near the front end optical components but will challenge the beam line designers to effectively utilize the longer length of beam line penetration in the shield wall. Additional advantages of the circular shield wall tunnel are that it's simpler to construct, allows greater access to the insertion devices and the upstream in tunnel beam line components, as well as reducing the volume of concrete and therefore the cost of the shield wall.

\footnotetext{
${ }^{1}$ This work is supported in part by the U.S. Department of Energy (DOE) under contract DE-AC02-98CH1-886.
} 


\section{Introduction}

The bulk shielding for the National Synchrotron Light Source-II (NSLS-II at Brookhaven National Lab.) accelerators was designed assuming a maximum operational beam loss power hitting a thick target in the vacuum chamber using the Analytic Shielding Model (ASM) [1-3]. The shield wall thickness transverse to the beam loss point was chosen to keep the dose rate less than the NSLS-II Shielding Policy [4] rate of $<0.5 \mathrm{mrem} / \mathrm{h}$ using the ASM estimates for the specified beam loss rate. Since the ASM dose rates were shown to be conservative values the measured dose rates tend to be less for these conditions. However abnormal beam losses frequently are not intercepted by thick targets but typically thinner vacuum chamber components, creating intense forward radiation showers. The most likely beam loss locations for the NSLS-II storage ring (SR) had supplemental shields (SS) designed to soften and attenuate this shower close to the loss point [5], such that the outer shield walls were more effective in reducing the radiation dose rate outside the tunnels. This was especially important for the $3 \mathrm{GeV}$ injection beam of the SR since most of the dose outside the tunnel is from neutrons.

While analyzing the SS designs for the NSLS-II SR, the model for the outer shield wall was initially simulated with a circular concrete shield, instead of the conventional saw-toothed ratchet wall design. When electron beam losses hit a beam line's relatively thin component (photon shutter or vacuum chamber flange), the conventional ratchet wall shows high levels of radiation outside the shield wall where the user beam line first optical enclosure (FOE) is located. This radiation can exists even when the safety shutters of the beam line are closed, allowing user access inside this FOE. During injections miss-steered beam could hit these beam line components creating significant radiation exposure for these users and staff. This is shown in Figure 1(a) for the FLUKA [6] estimated dose rates for $3 \mathrm{GeV}$ beam loss rate at $15 \mathrm{nA}$ a $10 \mathrm{~cm}$ copper target in the insertion device (ID) straight section of the NSLS-II storage ring tunnel (SRT). The SRT shows the intense forward radiation shower (Dose Eq. $>22 \mathrm{rem} / \mathrm{h}$ on beam plane) is shown exiting the thicker ratchet wall where the X-ray beam line would be extracted from tunnel. If the tunnel outer shield wall was replaced with a circular shield wall tunnel (CSWT) of $1 \mathrm{~m}$ thick concrete, the intense forward dose rate would not be observed (Dose Eq. $<1 \mathrm{mrem} / \mathrm{h}$ at the beam line extraction location) and the highest dose rate observed is transverse $\left(90^{\circ}\right)$ to the target, as shown in Figure 1(b). This forward dose rate outside the tunnels advantage is shown more clearly in Figure 2 where the lower dose rate is plotted with a linear scale, causing the in tunnel dose to be blacked out. The CSWT transverse (to the target) dose rate Fig. 2(b) is similar to the SRT dose rate $(\sim 60 \mathrm{mrem} / \mathrm{h})$ Fig. 2(a), since the distance and shield wall thickness are the same for this example. However as described below, the circular wall should be pulled further away from the ID beam chamber, in order to provide space and sufficient distance for the beam line front end components. The proposed $2.5 \mathrm{~m}$ distance of the outer wall to the ID beam chamber would reduce the transverse dose rate for the CSWT by a factor of 6.25 compared to the SRT dose rate.

\section{Circular Bulk Shield Wall Geometry}

The NSLS-II SR has a total circumference of $792 \mathrm{~m}$ made with 60 dipole magnets $\left(\theta_{b}=6^{\circ}\right.$ bend angle) having a bend radius of $25 \mathrm{~m}$. The beam vacuum chamber can be approximated by a circular orbit with and average radius $R_{b}=126 \mathrm{~m}$. Figure (3) shows the geometry of this average beam path along with a circular outer shield wall of radii: $R_{1}$ for the inner wall and $R_{2}$ the outer wall. Then the important quantities are the length of the X-ray beam line from the ID to the inner shield wall are: $L_{1}=R_{1} \bullet \sin \left(\theta_{1}\right)$ and the radiation path length through the shield wall $D_{2}=R_{2} \bullet \sin \left(\theta_{2}\right)-L_{1}$. For the NSLS-II SR the distance from the center of the long straight section ID to the conventional ratchet wall was $\sim 25 \mathrm{~m}$. To achieve this same distance $R_{1}=128.5 \mathrm{~m}$ or a transverse distance of $2.5 \mathrm{~m}$ 
from the beam chamber in the ID straight. This gives a transverse distance $2.5 \mathrm{X}$ the $1 \mathrm{~m}$ used in the Figure (1 and $2 \mathrm{~b}$ ) dose calculation, thereby reducing the transverse dose rate by the square of this distance increase. The outer concrete wall radius $R_{2}$ is just $R_{1}$ plus the wall thickness $t$. For the SRT $\mathrm{t}=1 \mathrm{~m}$ of concrete for the wall a distance of $1 \mathrm{~m}$ from the beam pipe[1]. This could be reduced by estimating the thickness using the ASM for the $2.5 \mathrm{~m}$ transverse distance but we use the $1 \mathrm{~m}$ thickness in order to be more conservative for the abnormal beam loss dose rates. For this example $R_{2}=129.5 \mathrm{~m}$ this gives the forward radiation path length of $D_{2}=4.7 \mathrm{~m}$. This is $3.35 \mathrm{X}$ the length of the NSLS-II ratchet wall thickness and why the forward dose rate is so much reduced. The ASM would estimate an attenuation for this forward dose of $>1250$ compared to NSLS-II. The forward dose reduction observed to be even greater $(\sim 22,000)$ than this estimate. However the NSLS-II ratchet wall actually had a lead SS installed in the ratchet wall concrete which reduces the dose rate an additional factor of $\sim 40$. This SS was meant to take the place of the Bremsstrahlung SS at the exit of the X-ray extraction port from the dipole. However this in wall SS was shown in [5] not to be as effective as placing the SS at the dipole exit and therefore the beam plane dose reduction is not as great as the ASM prediction, due to the secondary neutron production in the SS. Therefore all comparisons between the CSWT and the SRT will be made with the SS close to the beam loss location, but also includes the in shield wall SSs for the SRT model.

Since the potential forward dose reduction is so large and the transverse space between the beam line and the shield wall is small near the penetration, reducing beam length with penetration indentations inserted into the inner and outer circular shield wall is possible. The model for the CSWT is shown in Figure (4) with reduced the path length thickness by $\sim 1$ meter to $3.55 \mathrm{~m}$ with indentations sufficient to accept the safety shutters and the second Bremsstrahlung collimator. All other upstream components are further away from the shield wall and don't require indentations. This model kept the inner collimator distance from the ID center the same as for the NSLS-II damping wiggler beam line design. However if the outer collimator in the wall was the critical distance $\left(L_{2}\right)$ to maintain, then $R_{1}$ could be reduced by $\sim 0.8$ meter to keep $L_{2}$ the same distance as for NSLS-II damping wiggler beam line design. The geometrical parameters for the CSWT are listed in Eq. (1):

$$
\begin{gathered}
\theta_{1}=\cos ^{-1}\left(\frac{R_{b}}{R_{1}}\right) \quad, \quad \theta_{2}=\cos ^{-1}\left(\frac{R_{b}}{R_{2}}\right) \\
L_{1}=R_{1} \square \sin \left(\theta_{1}\right), L_{2}=R_{2} \square \sin \left(\theta_{2}\right), D_{2}=L_{2}-L_{1}
\end{gathered}
$$

and their dependence on $R_{1}$ for a constant concrete wall thickness of 1 meter, is shown in Figure (5). As the distance between the beam radius and the inner wall is reduced the effective beam radiation shielding thickness along the beam path increases.

The space between the X-ray beam pipe and the shield wall isn't large enough to place an access door next to the penetration wall, as was done in NSLS-II SRT. However that door placement was shown to have significant radiation issues for miss-steered beam that might hit X-ray beam line components [5]. The increased transverse distance between the beam line and the outer wall at the first dipole region is a better location for this access door. The forward radiation shower from the beam losses along the beam line would be less likely to cause radiation issues at this door location. The access door at this location can serve both the ID and BM front ends due in part to greater distance between the accelerator and the outer wall. In the present NSLS-II SRT the access door for the BM front ends are blocked by the FOE of the ID beam lines making it difficult to service the BM front ends. The placement of this door will require more detailed layout of the ID and bending magnet (BM) beam lines in the tunnel, in order to determine its best placement. The proposed layout of the door next to the first dipole is shown in Figure(6) for this CSWT model, which will make installation, re-alignment and maintenance easier. 


\section{Simulating the Radiation Levels for Abnormal Beam Losses}

The radiation shielding of the NSLS-II storage ring against miss-steered electron beam during injections was shown to pose the greatest risk outside the SRT[5,6]. Any place where that beam could hit thin material would create intense radiation at outside the tunnel. This required the beam to be intercepted with supplemental shields (SS) as close as possible to the beam loss point to effectively reduce the dose outside the SRT to acceptable levels. The X-ray extraction beam ports from the dipoles is the most probable of these thin material loss points and NSLS-II provided lead dipole supplement shields (DSS) after each dipole. These are shown in Figure 6 with the first DSS after the first dipole having a beam line vacuum pipe passing through it. The second dipole had a DSS further away from the dipole (due to space limitations from the quadrupoles and sextupoles) and doesn't have a beam pipe installed through it. These DSS's for uninstalled beam lines are most important, since the radiation shower off the vacuum flange and dipole beam power absorber isn't scattered or attenuated sufficiently and causes intense radiation dose outside the ratchet walls, even greater than shown in Figure 1(a) and 2(a). Therefore these DSS's are essential for the conventional SRT ratchet walls, while they are less important for the circular walls CSWT proposed here but will be included as a good shielding practice. These DSS have greater transverse distance to the shield walls for the CSWT than for the SRT case, which will reduce that dose rate for the same wall thickness or it could allow a thinner outer wall thickness to be used. It is more conservative and a good radiation practice to not reduce this wall thickness since the cost savings will be negligible.

For the CSWT penetration model shown in Figure 6, the radiation pattern and intensity outside the wall will be presented for the beam striking either of the two DSS's and compared with the SRT model. Other location will typically intercept more material (i.e. magnet yokes) and if they are thinner material will look like the loss patterns already simulated in Figures 1(b) and 2(b). The greatest advantage of the circular walls is there is never an orthogonal shield wall to any thin target beam loss direction as there is for conventional ratchet walls.

Miss-steered beam loss (always calculated for $3 \mathrm{GeV}$ beam with $15 \mathrm{nA}$ loss rate) from the second dipole hitting the solid DSS was the source for the high radiation levels outside the access doorways for the ratchet wall NSLS-II SRT[5,6]. The CSWT beam line penetration is near this DSS and the dose rate for beam hitting this DSS is shown in Figure 7. There are very low levels after the beam line penetration $(<3 \mathrm{mrem} / \mathrm{h}$ in the beam plane) and the greatest dose rates are (peak $<45$ $\mathrm{mrem} / \mathrm{h}$, of which $33 \mathrm{mrem} / \mathrm{h}$ is the neutron component) shown near where the access doors were located for the NSLS-II SRT. Figure 8 shows the dose rate outside the SRT for this same beam loss location. Figure 8(b) has a 4 times higher full scale dose rate than Figure 7(b). Outside the door the dose rate exceeds $200 \mathrm{mrem} / \mathrm{h}$ on the beam plane, which has only an $80 \mathrm{mrem} / \mathrm{h}$ neutron component (i.e. $60 \%$ gamma dose). However the Bremsstrahlung collimators and safety shutters attenuate the

beam plane dose and dose rate above their penumbra is $>800 \mathrm{mrem} / \mathrm{h}$, as shown in Figure 9(a). This is to be compared with the peak dose rate for the CSWT of $<48 \mathrm{mrem} / \mathrm{h}(33 \mathrm{mrem} / \mathrm{h}$ neutron component) on the beam plane with lower dose rates above the beam plane and no reduction on the beam plane, Figure 9(b). The asymmetry between the upper and lower dose levels for the CSWT arises from the offset of the DSS higher by $5 \mathrm{~cm}$, to attenuate the dose on the mezzanine above the beam line which has greater occupancy level. Similarly the dose rate inside the beam line FOE is considerably lower ( $>10$ times less) for the CSWT as compared to the SRT as shown in Figure 10.

The first DSS located after the first dipole of the lattice cell, will be most critical for the forward radiation dose from thin target beam losses for the SRT, as was shown in Figure 1 and 2. As shown for the CSWT this DSS is less critical due to the longer path length of the forward radiation shower in the shield wall and could be eliminated, without creating large dose rates in the FOE. This would actually be beneficial for the transverse radiation levels since the DSS would create a significant point loss as compared to a more distributed loss locations along the beam line 
components. However with the need to place an access door near this DSS loss location, we will analyze the beam loss on this DSS for both the SRT and CSWT models.

When miss-steered injection beam hits the first DSS in the SRT, the transverse scatter creates high levels $(>200 \mathrm{mrem} / \mathrm{h}$ ) near the lateral shield wall corner. This is partly due to distance and also because the wall was cut-off on the inside to allow better access at this tight area after the dipole. The dose distribution for this loss location in the SRT is shown in Figure 11, which also shows high levels at the transverse door location ahead of the BM beam line ratchet wall. This door way is filled with blocks of high density concrete that reduce the dose in the door opening but allow higher levels around the overlap between the low and high density concrete. The DSS attenuates the forward radiation shower 15meters upstream of the ID ratchet wall; however levels as high as $20 \mathrm{mrem} / \mathrm{h}$ are calculated above and below the beam plane in the FOE, which passes around the lead installed in the ratchet wall. On the beam plane the FOE dose levels are down to $\sim 2 \mathrm{mrem} / \mathrm{h}$ which is $>80 \%$ neutron dose.

Figure 12 show the same beam loss point at the first DSS in the CSWT model. As expected the location transverse to this DSS has the highest dose rates with significant levels outside the access door, which was estimated to be equivalent attenuation to the $1 \mathrm{~m}$ shield concrete shield wall by the ASM. The upstream end of the door has the high dose levels near $\sim 20 \mathrm{mrem} / \mathrm{h}$ as a consequence of the penetration through the overlap region between the door and the shield wall door jamb. This is from line of sight for the back scattered radiation off the DSS. In the FOE, the dose levels are below the calculated statistical level $(<0.01 \mathrm{mrem} / \mathrm{h})$.

\section{Designing Beam Lines for Circular Shield Walls}

The change from conventional ratchet wall designed beam lines to the CSWT beam lines will require some innovation by the beam line designers. Once the inner radius and wall thickness are determined all beam lines will have a fixed length from the dipole to the outer wall. The variation in length of the in tunnel distance would only vary with the ID length (two length defined for NSLS-II). However this is already fixed by the SRT to two ID beam lengths by the ratchet walls. The advantage of the CSWT is that as $R_{1}$ increases the penetration path length is reduces slightly (Fig.5), while the cost of the concrete increases only as $\Delta R_{1} / R_{1}$, and the dose rate decreases as $2 \Delta R_{1} / R_{1}$. Therefore the advantage of a greater distance to the outer wall can be a real benefit and can be accommodated with a larger aperture for the last penetration collimator in the shield wall. Having increased distance to the inner wall could allow other possible improvements in design and safety to be added, such as permanent magnet electron beam sweepers (Credited Safety Deviceused for a neutral hydrogen beam [7]) to be installed in the tunnel that would guarantee that Top-Off injection beam could never pass through the tunnel apertures to the FOE. This could eliminate the need for redundant safety shutter.

With the long path length in the shield wall yielding significant attenuation of the forward radiation, this could be reduced somewhat by increasing the outer indentation depth into the wall. This would have less of an impact than increasing the depth of the inner indentation, which becomes a secondary source of neutrons from the forward beam loss showers. This the source of the secondary peak dose observed transverse to the first penetration collimator in Figure 7(b). This level will increase if the inner indentation depth is increased. Another use of this long penetration might be to place a safety shutter within the shield wall penetration. This would require a new design for the out of vacuum NSLS-II safety shutter design since it might have more maintenance issues and potential vacuum leaks. If an in vacuum safety shutter was designed with simplified mechanical motion and lower potential failure rate, it could be installed in the wall, where access would be limited. 


\section{Architectural Design for Circular Shield Walls}

The architecture issues faced with outer circular shield walls has already been addressed for the inner shield walls for NSLS-II, which was generated by straight cord segments of $6.47 \mathrm{~m}$ subtending $\sim 3^{\circ}$ to approximate a circular inner shield wall. The distance from the beam chamber to the inner wall of the SRT is about $2.18 \mathrm{~m}$, which allows magnets girders and IDs to be transported from the inner service buildings, shown in the models. The outer lateral wall approaches 1 meter from the ID vacuum chamber. This distance is quite tight for the undulators in the SRT design, making the mechanics for the gap changing and supporting the magnetic forces quite restrictive and difficult to maintain. With larger access doors possible from the outside of the CSWT, out of vacuum undulators and wigglers could be changed from the outside of the ring without breaking vacuum. This would also allow larger mechanical supports to the outside of the ring where they would also provide some shielding for beam losses in the ID straight section. For in vacuum undulators (IVUs) the vacuum advantage is less important but access to the outer mechanics will still be easier for the CSWT.

The construction of the circular wall should be easier and therefore less expensive from the labor point of view. However from the comparison of the quantity of concrete, the CSWT outer walls described here represents about $11 \%$ less concrete in the outer walls than for the as built SRT (ignoring the roof concrete which is approximately the same area for both designs). Reducing the inner radius to $127.7 \mathrm{~m}$ from $128.5 \mathrm{~m}$, would allow the outer collimator to have the same distance as the present NSLS-II design, and would reduce that volume of outer wall concrete by less than $1 \%$ and the roof by a similar amount.

One significant advantage of the CSWT design is that the beam line penetrations, although initially fixed by the indentations formed during construction, could easily accommodate new beam line penetrations at previously unplanned locations. This flexibility could be a real advantage when future lattice upgrades are proposed for the existing tunnel, by allowing new beam source points to be provided with new penetrations. This would require boring through $\sim 4-5 \mathrm{~m}$ of concrete, but that is easier than reforming outer ratchet walls and the overhead roof concrete. One disadvantage of the CSWT design is that the length of beam lines from the dipole to the outer wall is fixed for all beam lines, while the total beam line length would only vary as the ID length ahead of the dipole varies. The ratchet wall model allows for different beam line length $\mathrm{s}$ as was done in the NSLS-II SRT.

\section{Summary and Conclusions}

A direct result of the NSLS-II effort to provide supplement shielding for the SR was the need to shield the ratchet walls and FOE from thin target beam losses and the high radiation levels they create. While studying these losses the property of natural shielding of these losses by a circular shield wall was rediscovered. This is a direct result of the beam loss directions never being orthogonal to a shield wall (ratchet wall) in the CSWT design. This zero ratchet wall design is contrary to the conventional design of light source shielding tunnels and should be reconsidered for future light source tunnels due to its greatly reduced radiation exposure to users and staff, in keeping with the ALARA principal requirement.

In summary, the CSWT shows orders of magnitude dose reduction for the thin target beam losses in Figure 1 and 2, as well as for the two DSS shielded beam losses in Figures 7-12 compared to those same loss locations for the SRT model. This arises from the fact that beam losses directions are never at right angles to the walls yielding a much greater attenuation length for the forward radiation shower generated by the beam loss. This property of the CSWT warrants further study to see if this reduction of radiation exposure is reasonable from the beam line design perspective, in keeping with the US Department of Energy directive for facility shielding to As Low as Reasonable Achievable (ALARA) levels [8]. 
The advantages of this CSWT design compared to the SRT are:

1) Greatly reduced dose rates in the forward beam loss direction and especially in the FOE.

This is due to the fact that circular walls never have orthogonal shield walls to any thin target beam loss direction as there is with conventional ratchet walls.

2) Reduced transverse dose rates from beam losses by increasing the distance to the outer shield wall without reducing the wall thickness. This has less cost impact than increasing this distance for the SRT design.

3) Outer tunnel access doors can be installed in locations away from the high forward radiation dose rates and will provide greater ease of use and access to two neighboring (ID and BM) beam lines.

4) The increased transverse distance to the IDs and upstream beam line components will make installation, realignment and maintenance easier.

5) Greater flexibility of the tunnel to changes in the beam extraction point and/or future uses of the tunnel for upgraded lattice designs.

6) Slightly lower cost for the volume of concrete needed in the tunnel walls and significantly lower cost of constructing the CSWT versus the SRT.

As with all things there are some disadvantages to be considered:

1) The beam line designers will need to reconsider the placement of their collimators and other beam line components in the tunnel.

2) Installation of the longer in wall collimators and shielding will be more difficult and require more effort.

\section{Acknowledgements}

This paper was the result of work by the Local Shielding Design Coordinating Group. This could not have been achieved without the continuing support and encouragement of F. Willeke, S. Sharma and S. Dierker. This work is supported in part by the U.S. Department of Energy (DOE) under contract No. DE-AC02-98CH1-886 


\section{Reference:}

[1] Panakkal K. Job and William R. Casey, Nucl. Instr. and Meth. A660, p. 1 (2011).

[2] W.P. Swanson, "Radiation Safety Aspects of the Operation of Linear Accelerators", IAEA Tech. Report 188 (1979).

[3] A.H. Sullivan, "A guide to Radiation near High Energy Particle Accelerators", Nuclear Technology Publishing (1992).

[4] NSLS-II Shielding Policy, PSD-C-ASD-POL-005 (2014).

[5] S.L. Kramer et. al., "Shielding Synchrotron Light Sources: Importance of Geometry for Calculating Radiation Levels from Beam Losses", submitted to Nuclear Inst. and Methods A (2015) and S.L. Kramer et. al., "Shielding Synchrotron Light Sources: Prediction and Verification of Radiation Levels from Electron Beam Losses", submitted to Nuclear Inst. and Methods A (2015). [6] FLUKA: a multi-particle transport code developed at CERN by Alfredo Ferrari, Paola R. Sala, Alberto Fasso and Johannes Ranft, https://www.fluka.org,

A. Ferrari, P.R. Sala, A. Fasso', and J. Ranft, "FLUKA: a multi-particle transport code" CERN2005-10 (2005), INFN/TC_05/11, SLAC-R-773.

[7] S.L. Kramer et. al., "A Low Cost/Low Intensity $50 \mathrm{MeV}$ Proton Irradiation Facility", PAC85, IEEE Trans. Nucl. Science 35, p. 3311 (1985).

[8] U.S. Department of Energy Directive, 10 CFR 835.1002 (Amended 2007), Facility design and modifications. 

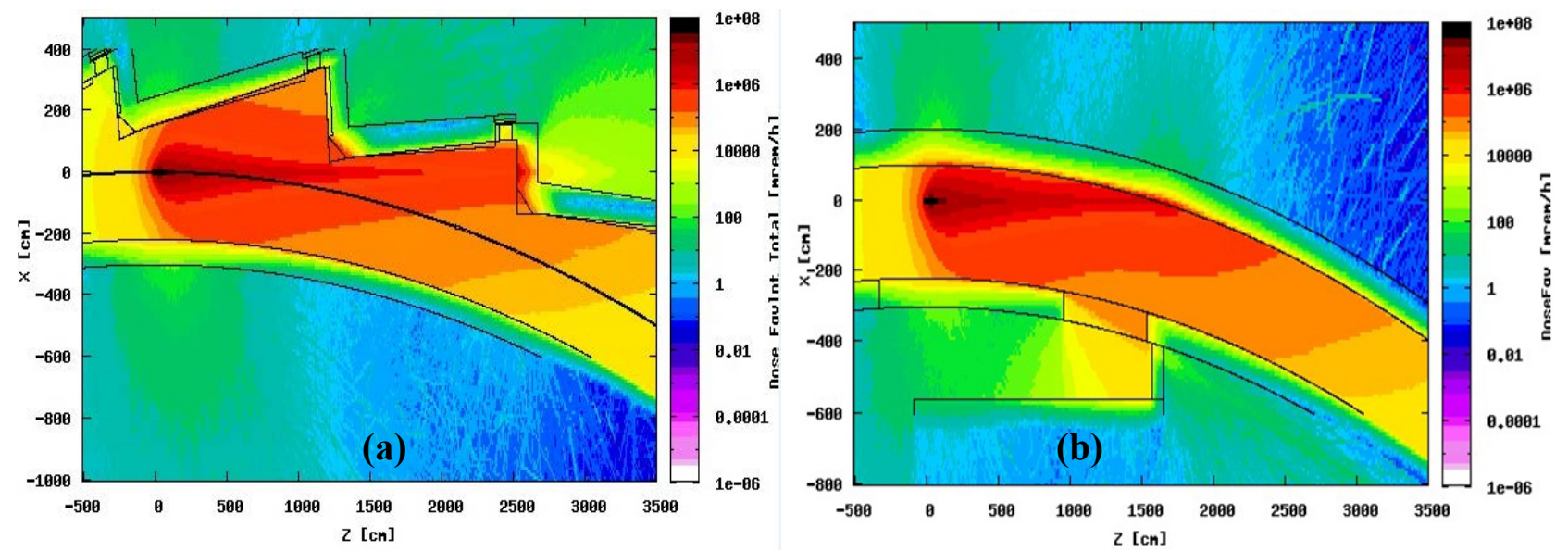

Figure 1 (a) The beam plane equivalent dose rate distribution (logarithmic dose scale) for a $3 \mathrm{GeV}$ beam loss rate of $15 \mathrm{nA}$ hitting a $10 \mathrm{~cm}$ copper target in the center of the ID straight section for SRT. (b) The equivalent dose rate for the same beam loss condition for a CSWT. No accelerator of beam line components are included.
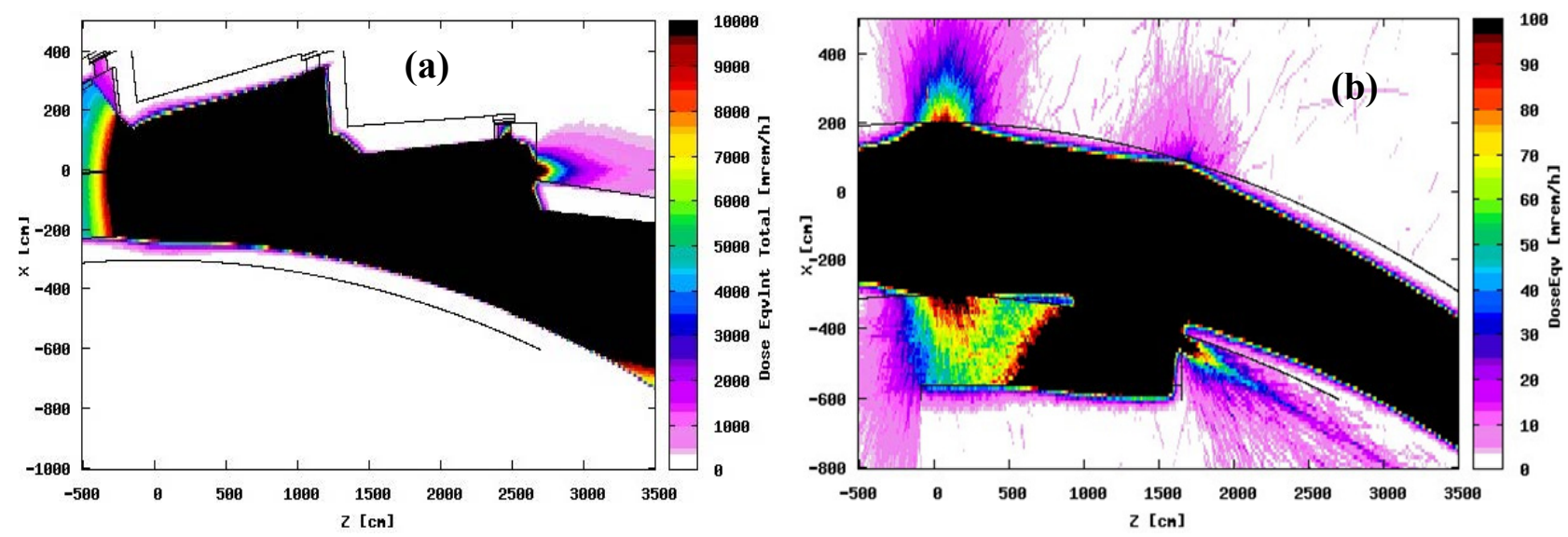

Figure 2 The same data as in Figure (1) but with linear dose rate scale with full scale of (a) 10rem/h and (b) $100 \mathrm{mrem} / \mathrm{h}$. This more clearly shows the dose rate outside the tunnel, with inner details blacked out. 


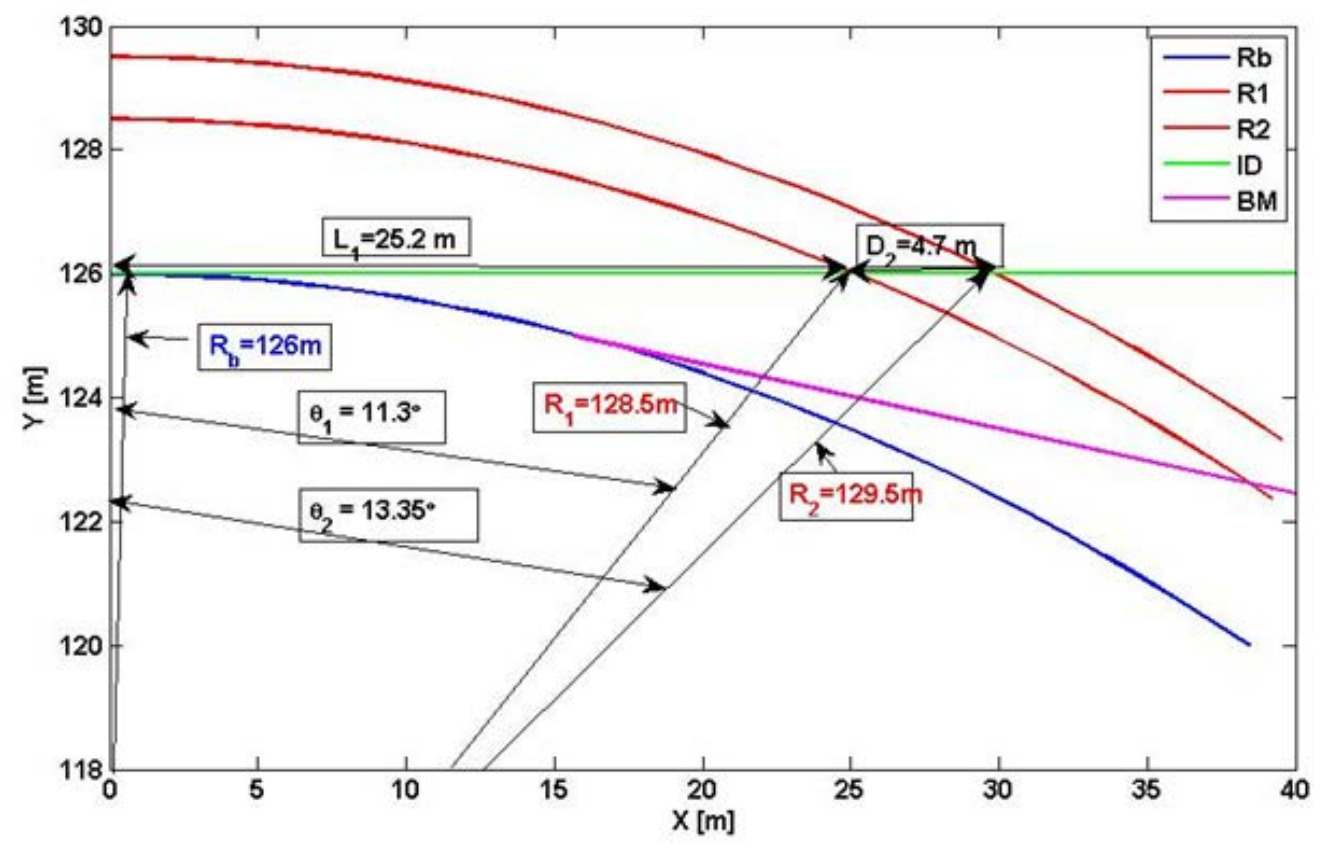

Figure 3 Geometry of a $1 \mathrm{~m}$ thick circular shield walls (red circles) for a given average beam vacuum pipe radius $R_{b}=126 \mathrm{~m}$ (blue). The beam lines are shown in green for the ID and magenta for the bending magnet $(\mathrm{BM})$.

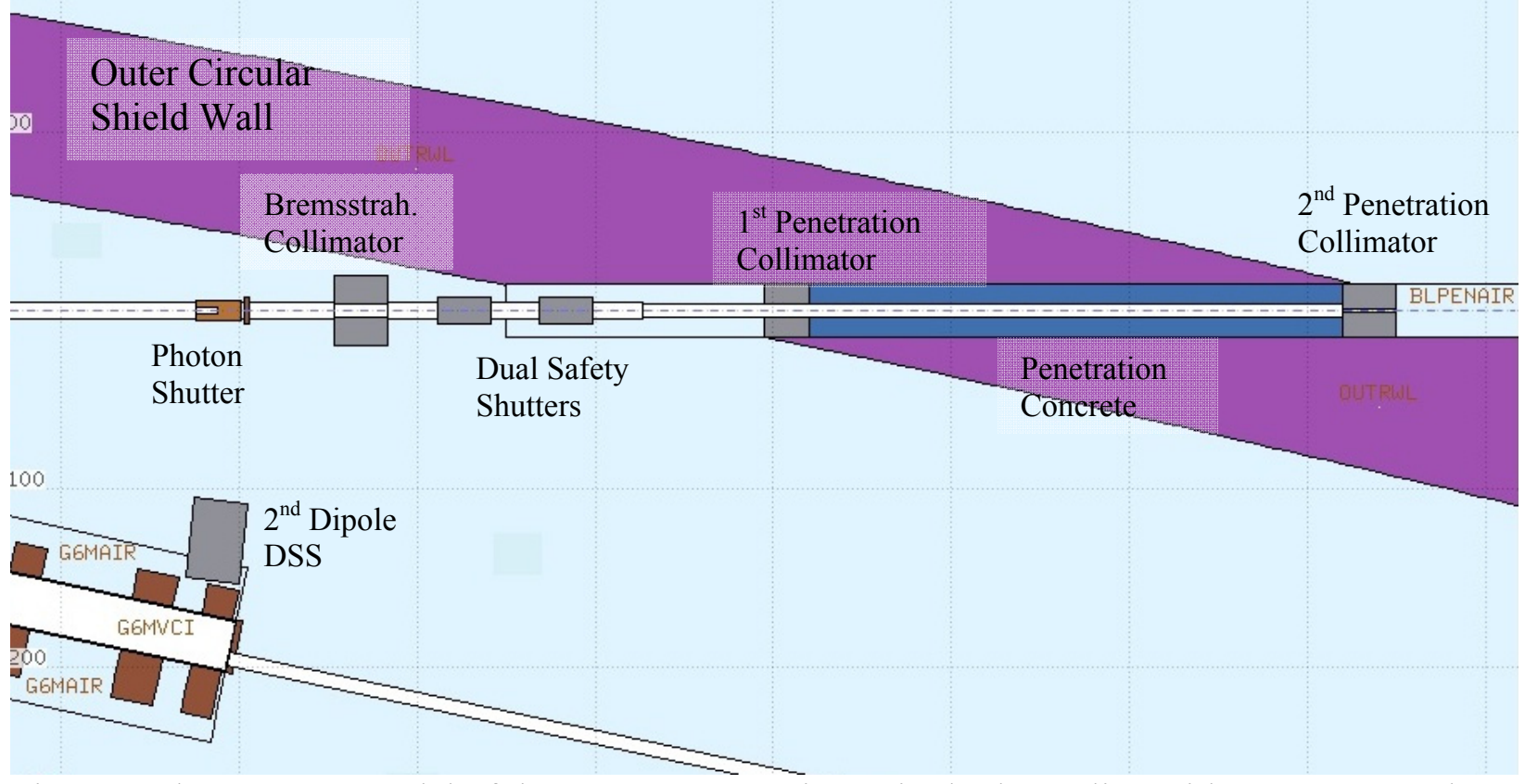

Figure 4 The FLUKA model of the NSLS-II Damping Wiggler beam line with components shown in the comparable CSWT model presented in Fig. (3). Smaller ratchet wall indentations are inserted in the shield wall to allow components to be installed upstream of the penetration, while reducing the radiation path length to $3.55 \mathrm{~m}$ of lower density concrete (dark green) plus Pb collimators (grey). 


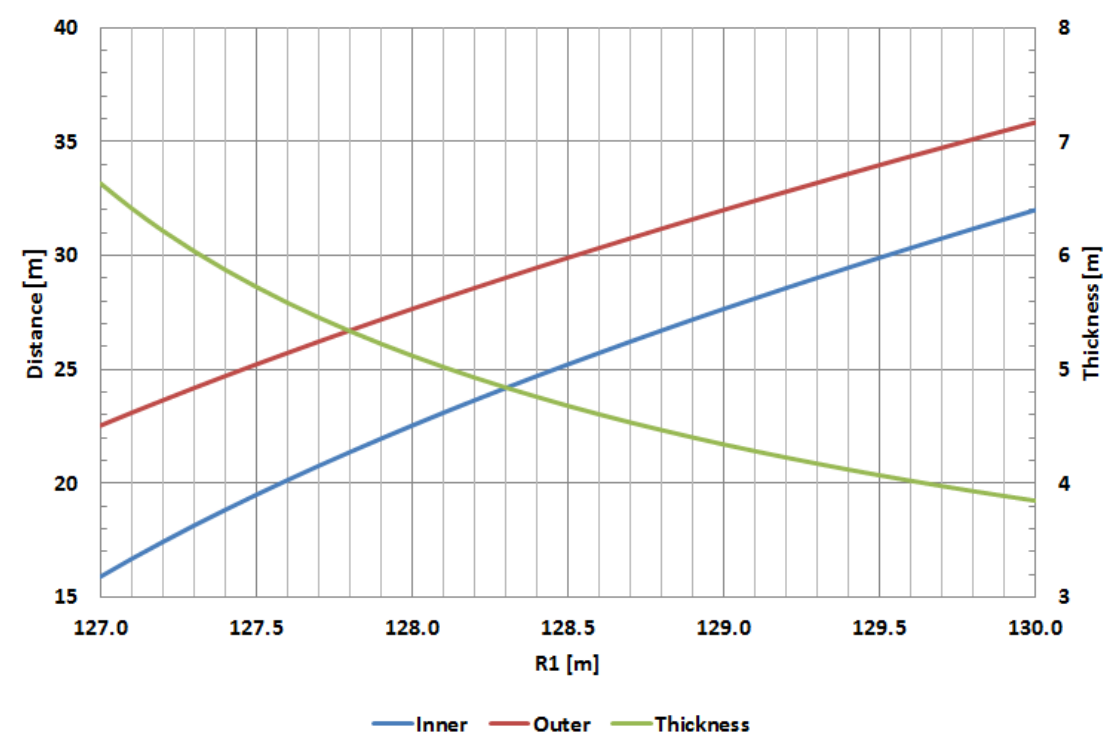

Figure 5 Shows the variation of distance from ID center to inner and outer edge of a 1 meter thick shield wall for $R_{b}=126 \mathrm{~m}$ beam radius versus the $R_{1}$ radius. The radiation effective shielding thickness $\left(D_{2}\right)$ through the shield wall in the forward beam direction is also shown.

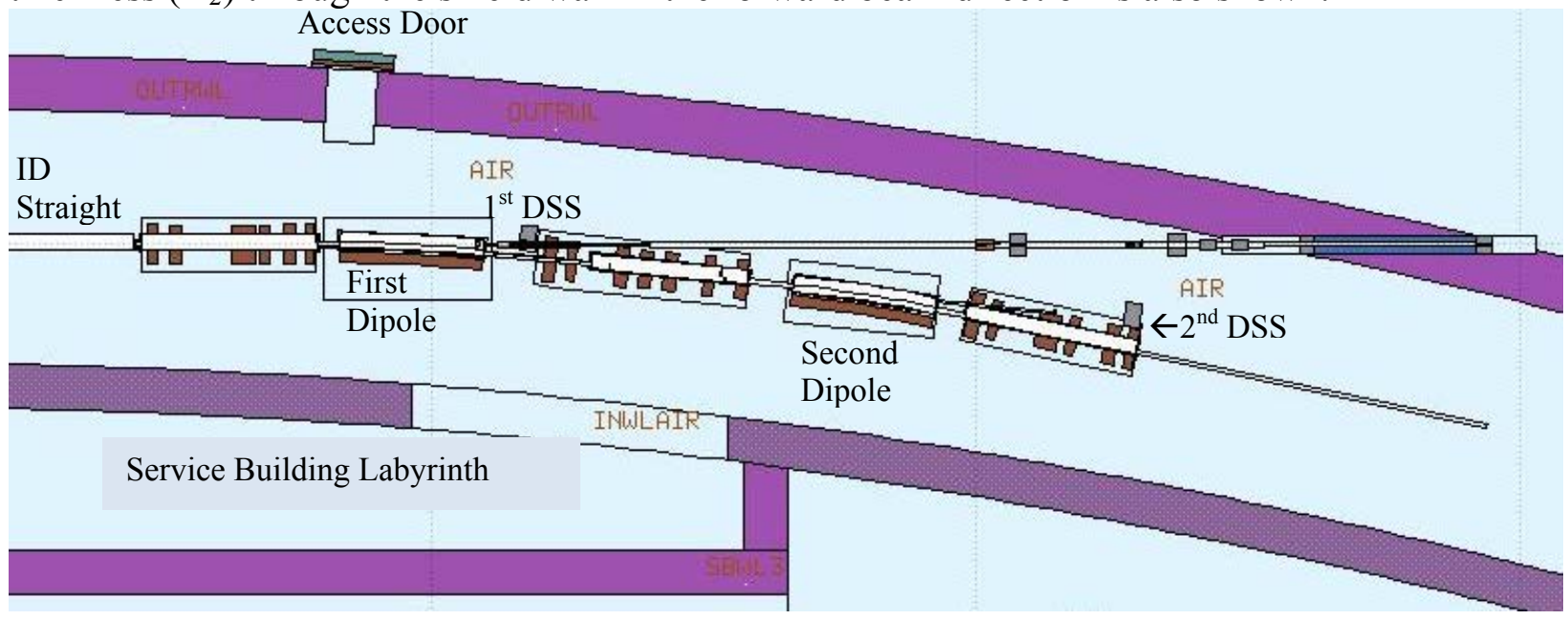

Figure 6 Show the NSLS-II SR accelerator components with the major damping wiggler beam line components in the CSWT with $R_{1}=128.5 \mathrm{~m}$. The sliding NSLS-II shield access door is shown adjacent to the first dipole. The beam line penetration has two $\mathrm{Pb}$ collimators and 3 meters of concrete blocks between them. The labyrinth is the access to the inner ring region through the service buildings. 

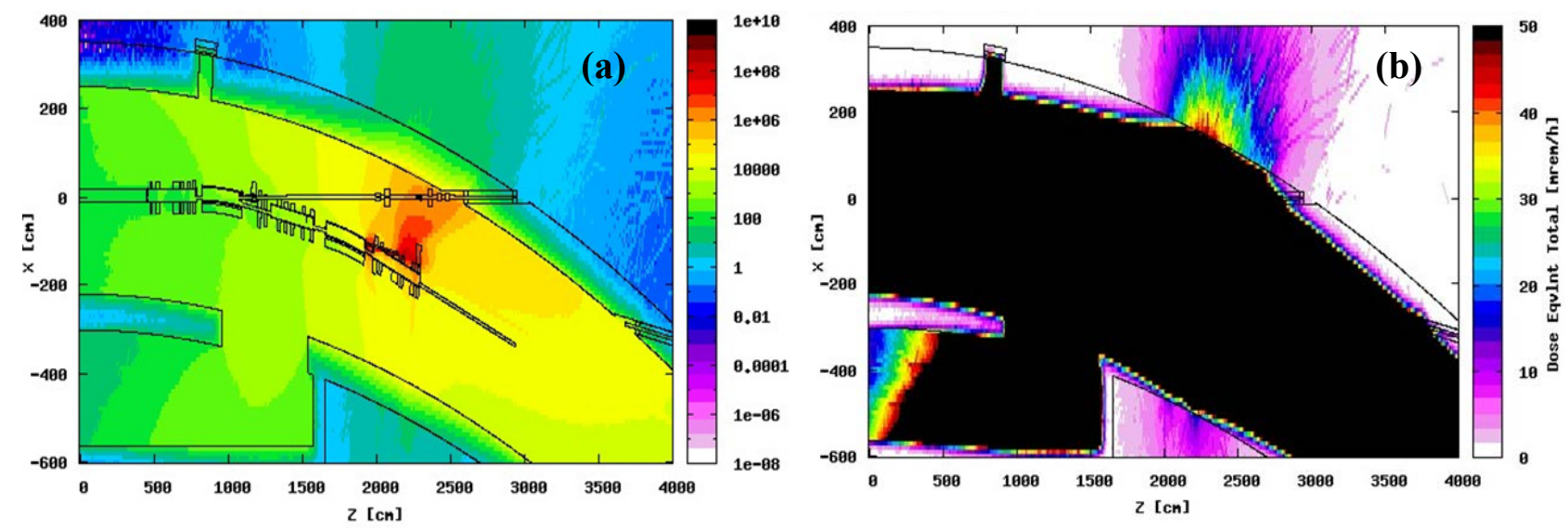

Figure 7 shows dose rate distribution for miss-steered beam hitting the second DSS after the second dipole for the CSWT model in Fig. 6. (a) shows the log scaled distribution in the tunnel and (b) linear with full scale $50 \mathrm{mrem} / \mathrm{h}$.
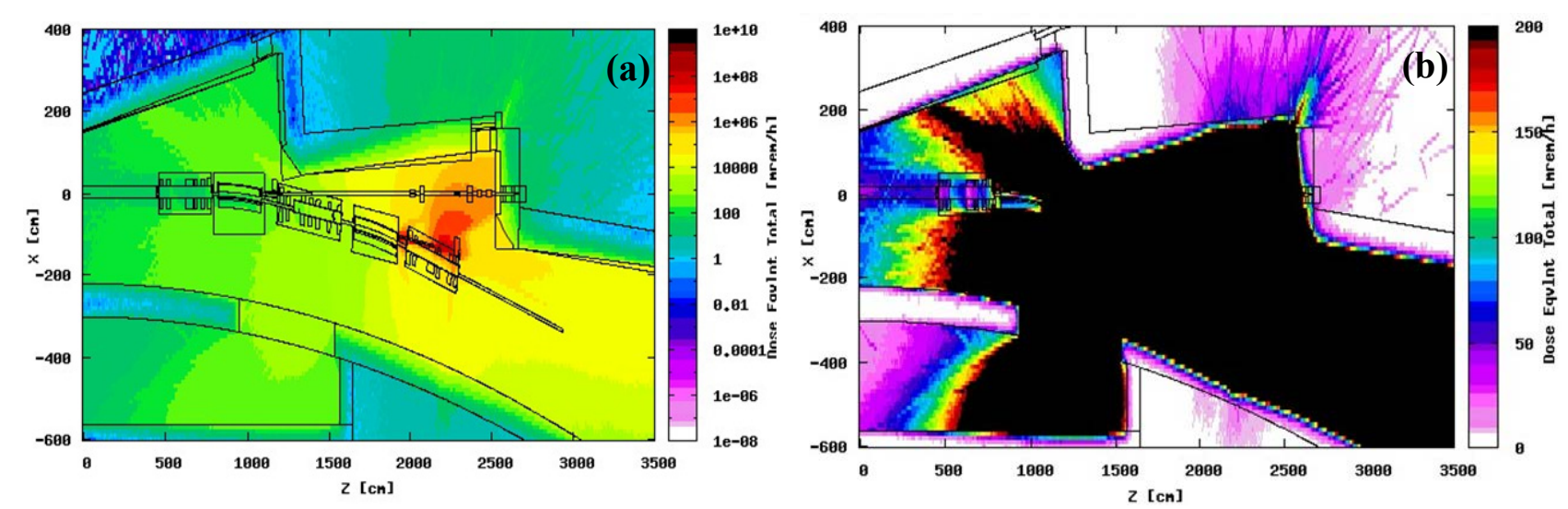

Figure 8 shows same beam loss location as in Figure 7 for the NSLS-II SRT ratchet walls. The scales for (a) are the same but (b) has a linear sacle of $200 \mathrm{mrem} / \mathrm{h}, 4$ times the $8(\mathrm{~b})$ scale.
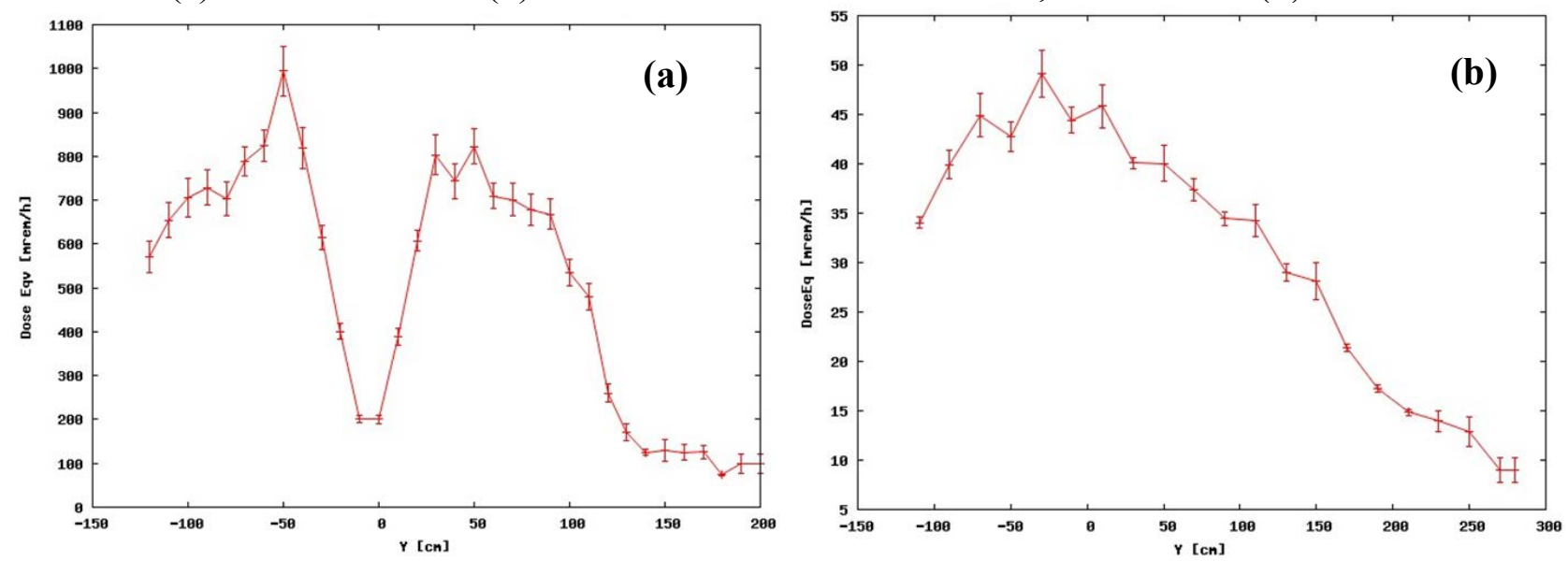

Figure 9 (a) Shows the vertical dose rate distribution at the peak door dose of the NSLS-II SRT in Fig. 8(b) and (b) shows the vertical dose rate distribution for the CSWT peak dose in Fig. 7(b) 

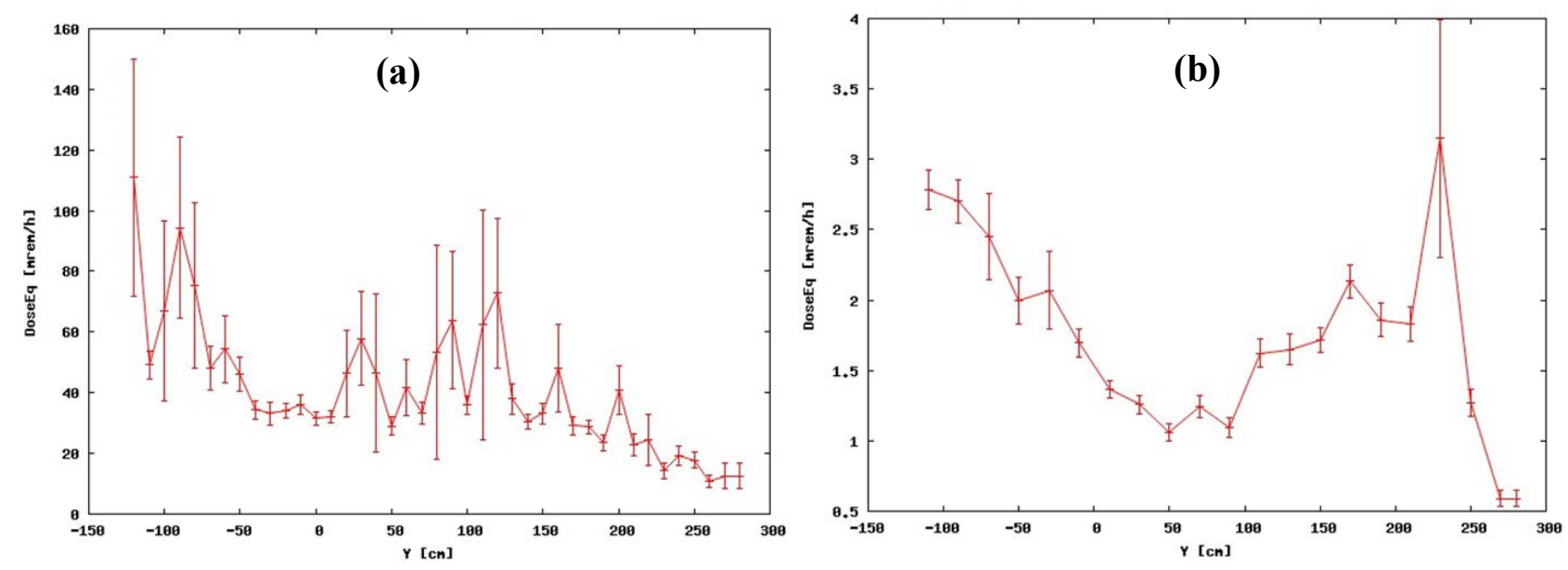

Figure 10 (a) Shows the vertical dose distribution, for the beam loss shown in Fig. 7 and 8, through the beam line penetration into the FOE for the NSLS-II SRT and (b) shows the same distribution for the CSWT.
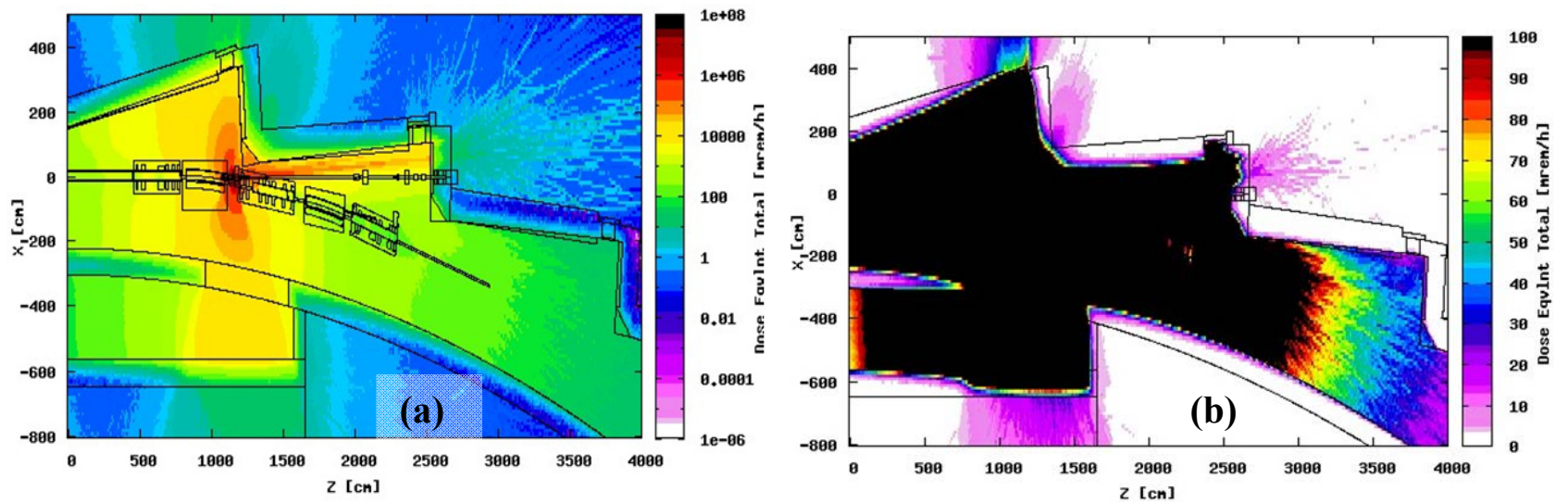

Figure 11(a) Shows the dose rate (logarithmic) for the NSLS-II SRT with beam hitting the first DSS and (b) for linear full scale dose rate of $100 \mathrm{mrem} / \mathrm{h}$.
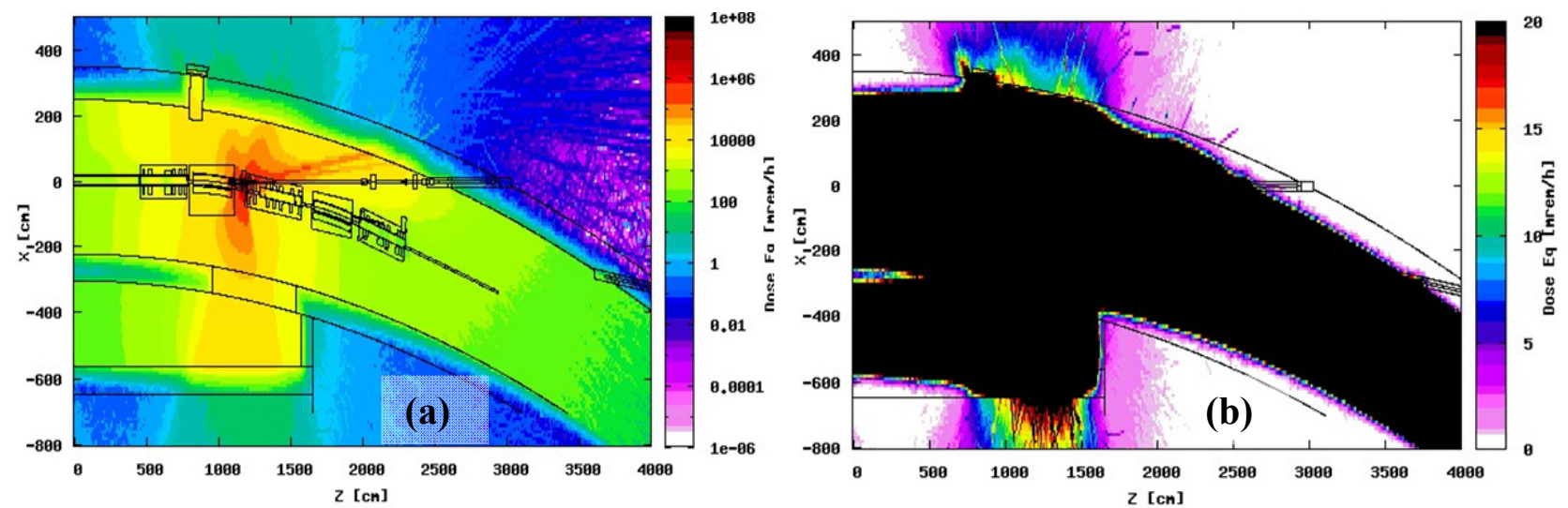

Figure 12 (a) Shows the dose rate (logarithmic) for the CSWT with beam hitting the first DSS and (b) for linear full scale dose rate of $20 \mathrm{mrem} / \mathrm{h}$. 